\title{
Management of hepatocellular carcinoma with novel immunotherapeutic agents and prospects for the future
}

\author{
Katarzyna Szklener ${ }^{1}$, Michał Piwoński², Klaudia Żak², Julita Szarpak², Sylwiusz Niedobylski², \\ Paweł Stanicki ${ }^{3}$, Sebastian Szklener ${ }^{4}$, Sławomir Mańdziuk ${ }^{1}$
}

\author{
'Department of Clinical Oncology and Chemotherapy, Medical University of Lublin, Lublin, Poland \\ ${ }_{2}^{2}$ Student Scientific Association at the Department of Clinical Oncology and Chemotherapy, Medical University of Lublin, Lublin, Poland \\ ${ }^{3}$ Student Scientific Association at the Department of Epidemiology and Clinical Research Methodology, Medical University of Lublin, Lublin, Poland \\ ${ }^{4}$ Department of Neurology, Medical University of Lublin, Lublin, Poland
}

The most frequent type of primary liver cancer is hepatocellular carcinoma (HCC). Although HCC is not the most frequent cancer, it is characterized by high mortality - the 5 -year survival rate is $6,9 \%$. In recent decades there was only one molecule available in treatment (sorafenib). However, in the past few years there have been advances in treatment. Nowadays, new generation tyrosine kinase inhibitors, check point inhibitors and anti-angiogenesis drugs are available. All those studies were analyzed outcome in context of monotherapy or combined therapies. In this review we made an attempt to compare results from different studies. Even though, many studies are undergoing final stages of clinical trials, it seems that combined therapies should be the next step in treatment advances.

Key words: hepatocellular carcinoma, targeted therapy, immunotherapy

\section{Introduction}

Hepatocellular carcinoma (HCC) is the most frequent type of primary liver cancer. Globally, each year approximately 750,000 new cases are diagnosed, so it constitutes $7 \%$ of all neoplasms [1]. Although HCC is not the most frequent cancer, it is characterized by high mortality - the 5 year survival rate is only 6,9\% [2]. Cirrhosis is the most important risk factor and is observed in $70-90 \%$ of patients [3].

Other factors are hepatitis B virus (HBV) infection, hepatitis $\mathrm{C}$ virus (HCV) infection, alcoholism and aflatoxin B1. While the exposition of $\mathrm{HBV}$ infection in high incidence areas appears in at least $50 \%$ cases of HCC, HCV infection is more common in lower incidence HCC areas like Eastern Europe and North
America $[4,5]$. It has also been suggested that non-alcoholic fatty liver disease (NAFLD), occurring as a consequence of obesity and diabetes, can be the cause of an increasing number of HCC cases [6].

The most widely used staging system for HCC is the Barcelona Clinic Liver Cancer algorithm (fig. 1). Cancer is classified as an early-stage when patients have single liver tumors or as many as 3 nodules measuring $3 \mathrm{~cm}$ or less. They are treated by resection, transplantation or ablation. Intermediate-stage cancer concerns greater tumor burden confined to the liver without any symptoms and chemoembolization can be a beneficial treatment method. Advanced-stage cancer is when HCC symptoms are present and/or extrahepatic cancer and/

\section{How to cite:}

Szklener K, Piwoński M, Żak K, Szarpak J, Niedobylski S, Stanicki P, Szklener S, Mańdziuk S. Management of hepatocellular carcinoma with novel immunotherapeutic agents and prospects for the future. NOWOTWORY J Oncol 2021; 71: 391-400.

This article is available in open access under Creative Common Attribution-Non-Commercial-No Derivatives 4.0 International (CC BY-NC-ND 4.0) license, allowing to download articles and share them with others as long as they credit the authors and the publisher, but without permission to change them in any way or use them commercially. 


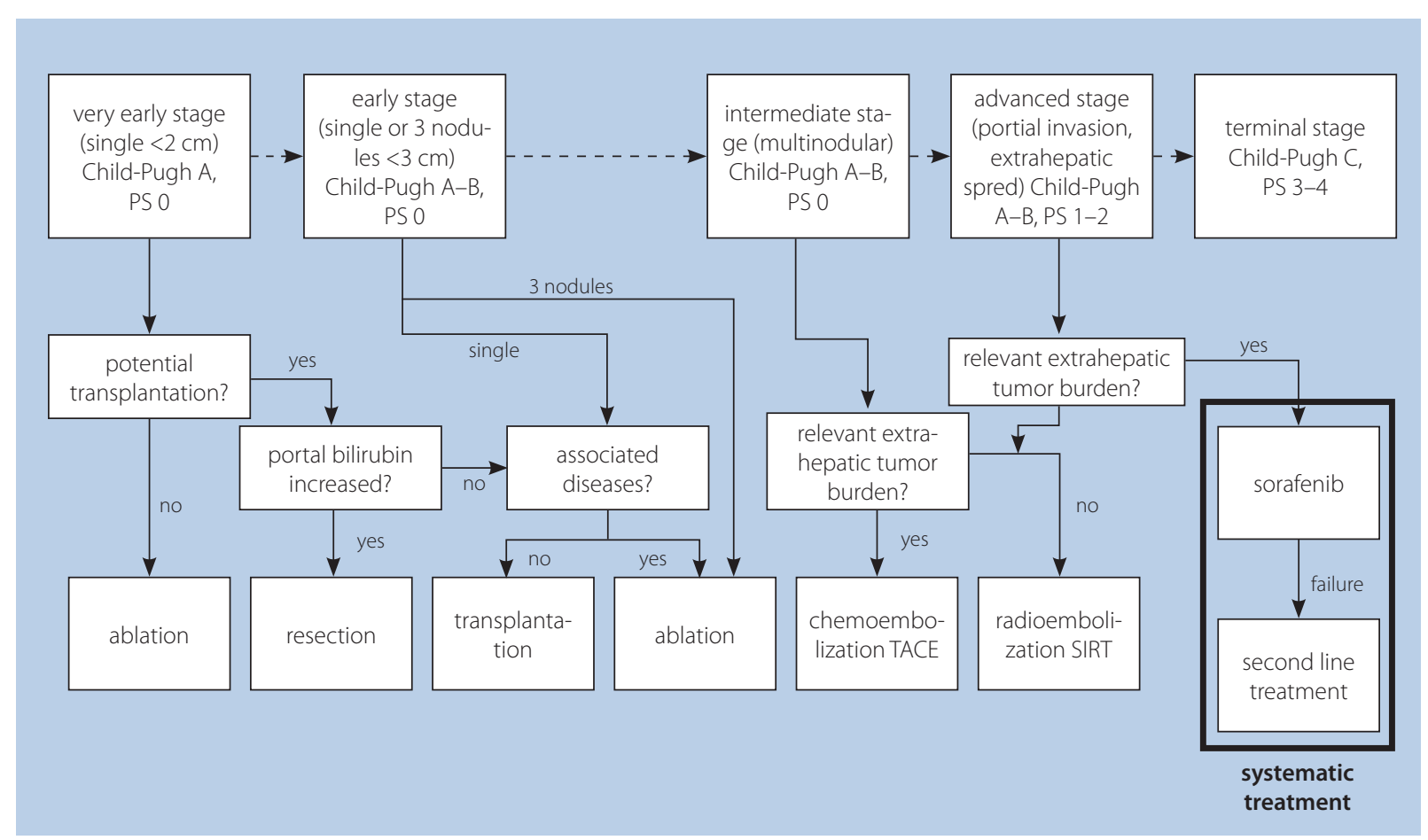

Figure 1. Barcelona Clinic Liver Cancer (BCLC) for HCC and available treatment strategies. In advanced stages, SIRT can be offered, if patients have no prognostically relevant tumor burden. Systemic therapy with sorafenib as a treatment by choice is recommended. In case of failure (clinical progression or intolerable toxicity), second-line treatment should be introduced [7]

or vascular invasion is/are diagnosed. The treatment of choice is the kinase inhibitor sorafenib [7].

\section{The use of multi-kinase inhibitors and anti- -angiogenic drugs in first-line treatment}

Sorafenib has been used in HCC treatment for more than a decade. It is also used in renal cell carcinoma (RCC) and differentiated thyroid cancer (DTC) [8]. The mechanism of action is based on the inhibition of the vascular endothelial growth factor receptor, the platelet-derived growth factor receptor (PDGFR) angiogenesis through targeting the mitogen-activated protein kinases/extracellular signal-regulated kinases (MAPK/ERK) pathway and receptor tyrosine kinases [9]. The role of sorafenib in HCC treatment is still being analyzed. The multicenter, double-blind, placebo-controlled trial of J.M. Llovet et al. focused on the differences in treatment of advanced HCC by sorafenib (at a dose of $400 \mathrm{mg}$ twice daily) and placebo. They found that the median overall survival (OS) rate was 10.7 months in the sorafenib group, and 7.9 months in the placebo group with no significant difference in the median time in symptomatic progression. The median time in radiologic progression was 2.7 months longer in the sorafenib group [10]. A similar result was presented by J. Bruix et al. as they found that sorafenib improved median OS and the disease control rate (DCR) compared to the control group [11]. Another study showed that the efficacy of hepatic arterial infusion chemotherapy (HAIC) with cisplatin followed by sorafenib does not improve the survival rate in comparison with sorafenib alone; the median OS period in the HAIC group was 10 months and in the sorafenib group 15.2 months [12].

Lenvatinib is another drug used as a first-line treatment of HCC. Its mechanism is based on an inhibition of multiple receptor tyrosine kinases, including the vascular endothelial growth factor receptor 1 (VEGFR1), the vascular endothelial growth factor receptor 2 (VEGFR2) and the vascular endothelial growth factor receptor 3 (VEGFR3). It also impacts on angiogenesis, tumor growth and cancer progression by fibroblast growth factor receptors: FGFR1, FGFR2, FGFR3, FGFR4 and the platelet derived growth factor receptor alpha (PDGFRa) inhibition. Although there is no comprehensive comparison of both drugs in terms of OS, lenvatinib is considered as an alternative for sorafenib as there is significant improvement in OS (lenvatinib - 13.6 [95\% Cl: 12.1-14.9] months vs. sorafenib - 12.3 [95\% Cl: 0.4-13.9] months), longer progression-free survival (PFS) (7.4 [95\% Cl: 6.9-8.8] vs. 3.7 [95\% Cl: 3.6-4.6]) and time to progression (TTP) (8.9 [95\% Cl: 7.4-9.2] vs. 3.7 [95\% Cl: 3.6-5.4]) [13].

\section{Second-line treatment}

It is estimated that up to one-third of patients with advanced HCC qualify for second-line therapy. The necessity to change treatment options results from the failure of first-line therapeutics due to their high toxicity, disease progression or resistance to therapy of primary or adaptive mechanisms [14]. A study by Fung et al. on 730 Canadian patients showed that only $13.1 \%$ of patients would qualify for second-line treatment with 
regorafenib, cabozantinib or ramucirumab after using strict eligibility criteria (SEC). In turn, applying modified eligibility criteria (MEC) increased the size of the group under therapeutic treatment by more than half, reaching 31.7\% [15]. Tivantinib, brivanib, and everolimus were considered promising candidates for inclusion as second-line systemic therapy for HCC. Unfortunately, in the third phase of clinical trials, they did not show any significant benefit in terms of OS compared to the placebo [16-18].

\section{Regorafenib}

Until recently, patients treated with sorafenib who had not yet completed therapy due to progression or tolerance, could not count on any alternative form of systemic treatment. Bruix et al. (2016) published the results of their RESORCE study. They proved the effectiveness of using regorafenib as a second-line treatment in patients previously treated with sorafenib [19]. Regorafenib is an orally administered inhibitor of a set of multiple kinases responsible for angiogenesis (including vascular endothelial growth factor receptors 1-3 (VEGFR 1-3), tyrosine kinase with immunoglobulin-like and epidermal growth factor-like domains 2 (TIE2), fibroblast growth factor receptors 1-2 (FGFRs 1-2), the formation of metastases [VEGFR 2-3, PDGFR]) or the development of tumor immunity (colony-stimulating factor-1 receptor [CSF-1R]). These processes play a crucial role in the development of cancer and its progression [20]. In addition to second-line treatment, it is also used in therapy of refractory metastatic colorectal cancer ( $\mathrm{mCRC}$ ) and advanced gastrointestinal stromal tumors (GIST) $[20,21]$.

\section{Cabozantinib}

Cabozantinib is an orally administered tyrosine kinase inhibitor [TKI]. Its scope of action includes antagonistic effects against VEGFR 2, MET, KIT, RET, and AXL [22]. The MET tyrosine kinase receptor is the receptor for the hepatocyte growth factor (HGF). Their cooperation in a physiologically developing organism is important for processes such as the proper proliferation of cells or their motility [23]. However, in the case of HCC, melanoma, pancreatic, prostate, or ovarian tumors this mechanism is used by the tumor for its own benefit including growth and spread $[22,24]$. HGF and MET antagonism, in turn, results in inhibition of tumor growth [25]. It is also believed that targeted MET and HGF therapies can overcome the barrier of HCC resistance to sorafenib treatment [26].

Based on the phase 3 results of the CELESTIAL study, cabozantinib was included in second-line standard of care for patients who had previously received sorafenib and had progressed. Abou-Alfa et al. conducted a randomized double-blinded trial including 707 patients with advanced HCC. The group was divided in a 2:1 ratio and the majority of patients received cabozantinib orally while the rest of the group received placebo. The initial dose was $60 \mathrm{mg}$ and was decreased successively to $40 \mathrm{mg}$ and $20 \mathrm{mg}$ if necessary due to adverse events. The primary endpoint was OS while the secondary end points were PFS and objective ORR. Cabozantinib significantly increased the median of OS compared to placebo (respectively 10.2 months for cabozantinib and 8.0 months for placebo; HR 0.76; 95\% Cl: 0.63-0.92; $p=0.005$ ). The median PFS was 5.2 months for cabozantinib and 1.9 months for placebo ( $\mathrm{HR} 0.44$; $95 \%$ Cl: 0.36-0.52; $p<0.001)$. Among the adverse events during therapy, the most frequent were palmar-plantar erythrodysesthesia (17\% with cabozantinib and $0 \%$ in the placebo group), hypertension (16\% and $2 \%$, respectively), increased aspartate aminotransferase level (12\% and $7 \%$, respectively), fatigue (10\% and $4 \%$ ), and diarrhea (10\% and $2 \%$ respectively) [27].

\section{Ramucirumab}

Ramucirumab is a recombinant human lgG1 monoclonal antibody that impairs angiogenesis which is essential for tumor development due to its VEGFR 2 antagonistic activity [28]. In a randomized double-blinded third phase REACH trial by Zhu et al., the efficacy of ramucirumab compared to a placebo was determined in patients with advanced HCC who had previously received sorafenib treatment. Initially there was no significant improvement in OS in ramucirumab-treated patients compared to placebo. Median OS of ramucirumab was 9.2 months vs 7.6 months with placebo administration (95\% Cl: 0.717-1.046; $p=0.1391$ ) [29]. However, subsequent data analysis showed that ramucirumab significantly improved the OS score in patients with a-fetoprotein (AFP) $\geq 400 \mathrm{ng} / \mathrm{mL}$ [30]. The REACH II study included patients with advanced HCC (AFP values $\geq 400 \mathrm{ng} / \mathrm{mL}$ ) and Child-Pugh class A liver disease treated only with sorafenib. 197 out of 292 subjects received ramucirumab therapy at a dose of $8 \mathrm{mg} / \mathrm{kg}$ intravenously every 14 days while 95 of them received placebo. The results showed that the median OS of ramucirumab treated patients was 8.5 months vs 7.3 months in the placebo group (HR of 0.71 [95\% Cl: 0.53-0.95]) while the PFS for ramucirumab was 2.8 months vs. 1.6 months for placebo ( $p<0.0001)$. The most common adverse effects included hypertension (13\% with ramucirumab vs. $5 \%$ with placebo), hyponatremia ( $6 \%$ vs. $0 \%$, respectively), and increased aspartate aminotransferase (3\% vs. 5\%, respectively) [31].

\section{Immunotherapeutic agents}

One of the major problems in tumor management is their ability to escape from the immune system's range of action. Immunoediting, which is a key aspect of immune evasion, is based on tumor-immune system interactions and Darwinian selection leading to decreased immunogenicity of the neoplastic cells. This in turn makes them invulnerable to the immune response. Attempts have been made to counteract those effects with immunotherapy. Experimental immunotherapy consists of two approaches: inducement of a new immune response and enhancement of the existing one [32]. Strategies of the de novo response stimulation include the usage of antigen targeting 
antibodies coupled with the immune cells, e.g. anti glypican 3 antibodies [33] and anti alpha-fetoprotein antibodies [34] conjugated with T cells or NK cells. Other examples are adoptive cell therapy using the chimeric antigen receptor expressing $T$ cells (CAR-T cells) [35], cytokine induced killer cells (CIK cells) [36] or natural killer cells (NK cells) [37] and vaccine therapies with dendritic cell vaccines [38] or peptide vaccines [39].

The reinforcement of the existing immune response is based mainly on the pre-existing reactivity to neoplastic cells impeded by the microenvironmental components of the immune-edited tumor. One of the techniques aims at immune-inhibitory cytokines such as transforming growth factor beta (TGF- $\beta$ ) secreted by the neoplasm. The most relevant approach though, nowadays, is connected with the inhibition of immune checkpoints which is crucial in cancer immune evasion processes $[40,41]$.

Programmed death receptor 1 (PD1) is a surface protein expressed mainly by Tc lymphocytes but also by Th lymphocytes, Treg lymphocytes, B lymphocytes, NK cells, and some myeloid cells [32,33] which binds with its ligand PD-L1 (programmed cell death ligand 1). This triggers the metabolic cascade resulting in the inhibition of immune response by increasing the number and activity of Treg cells [42], inactivation of CD28 and downregulation of TCR in Tc cells [43] or their apoptosis $[42,44]$. There are a number of clinical trials of PD1 inhibitors.

\section{Nivolumab}

Nivolumab is used for the treatment of patients with confirmed HCC and previously unsuccessfully treated with sorafenib. This indication was approved by the U.S. FDA in 2014 under accelerated approval due to its high efficacy and manageable safety profile demonstrated in CheckMate 040 open-label non-comparative phase $1 / 2$ dose escalation and expansion trial in advanced HCC [45]. During both dose-escalation $(n=48)$ and dose-expansion ( $n=214$ ) phases ( $3 \mathrm{mg} / \mathrm{kg}$ ), nivolumab showed acceptable tolerability. Although 46 of the dose-escalation patients (96\%) discontinued treatment, 42 cases (88\%) were due to disease progression. In the dose-expansion phase, the objective response (assessed using RECIST 1.1) was 20\% (95\% Cl: 15-26) and in the dose-escalation phase it turned out to be 15\% (95\% Cl: 6-28). The median OS rate was about two months longer (16.39 months) compared with sorafenib-treated patients (14.69 months). CheckMate 459, a phase III study on nivolumab in HCC (NCT02576509) is already in progress and results have not been published yet [46].

\section{Camrelizumab}

Camrelizumab is a humanized high-affinity lgG ${ }_{4}$-kappa antibody PD-1 inhibitor used for the treatment of various neoplasms [47]. It has already received its first conditional approval in China for relapsed or refractory classical Hodgkin's lymphoma treatment in patients after receiving at least two systemic chemotherapies. Its safety and efficacy in patients with pre- treated advanced HCC was evaluated in a multicenter open-label parallel-group and randomized phase 2 clinical trial [48]. The objective of the partial response evaluated by blinded independent central review (BICR) according to RECIST 1.1 was achieved in 32 of 217 patients (14,7\%; 95\% Cl: 10.3-20.2). The 6-month OS probability was 74,4\% (95\% Cl: 68-79.7), the 12-month OS was 55.9\% (95\% Cl: 48.9-62.2) and the median OS turned out to be 13.8 months (95\% Cl: 11.5-16.6). The rate of treatment-related adverse events was relatively low and manageable (grade 3 or 4 in 22\% of patients). Phase III studies on camrelizumab in HCC (NCT03605706) and other malignancies such as non-smalll-cell lung carcinoma (NSCLC), gastric / oesophageal cancer, nasopharyngeal carcinoma are pending or ongoing [49].

\section{Pembrolizumab}

Pembrolizumab is a humanized monoclonal lgG4 kappa antibody that acts as a PD1 inhibitor indicated for a variety of neoplasms besides HCC, such as melanomas, NSCLCs, head and neck squamous cell carcinomas (HNSCCS), several type of lymphomas and others. The usage of pembrolizumab in HCC was approved by the FDA under accelerated approval based on tumor response rate and durability of the response shown in the KEYNOTE-240 study [50]. KEYNOTE-224 is a single-arm non-randomized multicenter open-label phase 2 trial [51] on 104 patients after disease progression, on or after sorafenib therapy and who had measurable disease and Child-Pugh class A liver impairment. 18 of the 104 patients displayed an objective response (17\%; 95\% Cl: 11-26) including 1 complete and 17 partial responses. Treatment-related adverse events of grade 3 or worse were reported in 27 (26\%) participants and grade 4 and grade 5 events affected 1 patient each. The results of further assessment in phase III trial KEYNOTE-240 were consistent with those of KEYNOTE-224, although OS and PFS measurements did not reach their co-primary endpoints and statistical significance per specified criteria. There are a number of trials studying other PD-1 inhibitors such as sintilimab (NCT03794440) [52], or tislelizumab (NCT03412773) [53]. Meta-analysis by Voutsadakis carried out with trials of selected PD-1 inhibitors showed no dissimilarities in effectiveness with other systemic therapies for HCC [54].

\section{Durvalumab}

Durvalumab is a monoclonal human immunoglobulin G1 kappa. The safety and efficacy of this PD-L1 inhibitor in relation to HCC was assessed in phase 1/2, a multicenter open-label study in patients with advanced solid tumors (NCT01693562). Grade 3-4 treatment-related adverse events occurred in 20\% of patients and antitumor activity measured in ORR was calculated to be $10.3 \%$ (95\% Cl: 2.9-24.2) with a median OS of 13.2 months (95\% Cl: 6.3-21.1) [55].

Cytotoxic T-lymphocyte-associated protein 4 (CTLA4) is another receptor that acts as an important immune checkpoint 
that through various mechanisms (e.g. inhibition of cytotoxic T lymphocytes, dendritic cells, activation of regulatory $T$ cells etc. $[56,57])$ contributes to the tumor immune evasion. The only CTLA4-inhibitor tested on HCC patients was tremelimumab - the results of the phase I trial displayed a partial response rate of $17.6 \%$ without major safety concerns [58]. HIMALAYA, a phase III study on tremelimumab in HCC (NCT03298451), is already in progress as well [59]. Other immune checkpoints potentially relevant in HCC therapy (and oncology in general) are TGF-beta (e.g. NIS793 with PD-1 inhibitor spartalizumab NCT02947165) [60], T-cell immunoglobulin and mucin-domain containing-3 (TIM-3) (e.g. anti-TIM-3 and PD-1 - NCT03680508) [61] or lymphocyte activation gene 3 (LAG-3) (e.g. relatlimab and nivolumab - NCT01968109) [62].

\section{Tremelimumab}

As for other immunotherapeutics, with regards to worth mentioning is the combination of tremelimumab which is a human monoclonal antibody anti-CTLA-4 with ablative therapies that tend to induce peripheral immune responses [63-65]. According to a clinical trial by Duffy et al., in 19 patients who underwent tremelimumab administration and subtotal radiofrequency ablation or chemoablation, a confirmed partial response was observed in five subjects (26.3\%; 95\% Cl: 9.1-51.2). Those patients received the infusion at two dose levels (3.5 and $10 \mathrm{mg} / \mathrm{kg}$ i.v.) given every 4 weeks for a total of 6 doses followed by 3-monthly administrations until the fulfillment of off-treatment criteria. In the study group, the median PFS was 7.4 months ( $95 \%$ Cl: 4.7-19.4 months) and OS - 12.3 months (95\% Cl: 9.3-15.4 months) [66]. What is more, tremelimumab shows a significant activity in patients with HCC and chronic HCV infection and these conjectures were confirmed both in the study mentioned above [66], but also in the results of the clinical trial of Sangro et al. In the second case given, no ablative treatment was used and also the drug dose was different $-15 \mathrm{mg} / \mathrm{kg}$ i.v. every 90 days until progression or toxicity occurrence. Median TTP was 6.48 months (95\% Cl: $3.95-9.14$ ) and a significant drop in the viral load provoked by the anti-HCV increased immune response was confirmed [58].

\section{Combined therapies}

Studies are underway to show the efficacy of a combination of anti-cancer drugs in the treatment of HCC. Combinations of anti-angiogenesis drugs with inhibitors of immune checkpoints (PD-1, PD-L1) used primarily in the treatment of lung cancer are being tested.

\section{Atezolizumab and bevacizumab}

In recent months, anti-PD-L1 activity has gained prominence in HCC treatment due to its anti-proliferative activity. The aim of the GO30140 study was to investigate the significance of atezolizumab (anti-PD-L1) alone and in combination with bevacizumab (anti-VEGF) in unresectable HCC. In a group of patients receiving the combined therapy of atezolizumab (1200 mg) and bevacizumab (15 mg/kg) with a median follow-up of 12.4 months, a confirmed objective response was observed in 37 (36\%; 95\% Cl: 26-46) out of 104 patients; while for PFS, with a median follow-up of 6,6 months, it was 5.6 months (95\% Cl: 3.6-7.4). In the group receiving only atezolizumab in monotherapy, the median PFS was 3.4 months (1.9-5.2; hazard ratio $0.55 ; 80 \% \mathrm{Cl}: 0.40-0.74 ; \mathrm{p}=0.011)$ [67]. Hack et al. compared also the HR for death -0.58 (95\% Cl: 0.42-0.79; $\mathrm{p}<0.001)$, OS at 12 months being $67.2 \%$ (95\% Cl: 61.3-73.1) vs. $54.6 \%(95 \% \mathrm{Cl}: 45.2-64.0)$ and PFS - 6.8 months (95\% Cl: 5.7-8.3) vs. 4.3 months (95\% Cl: 4.0-5.6); this was in atezolizumab plus bevacizumab and sorafenib-receiving groups with unresectable HCC with no prior systemic treatment. The results showed significantly better OS and PFS outcomes in atezolizumab + bevacizumab than sorafenib-receiving patients. According to the NCT04102098 study results, the PFS (in months) in the atezolizumab and bevacizumab group was even longer - 6.8 (5.7-8.3) [68].

\section{Nivolumab and ipilimumab}

As for other immunotherapeutic possibilities, there is much more to discover. In a Checkmate 040 randomized clinical trial in group A (obtaining nivolumab $1 \mathrm{mg} / \mathrm{kg}$ with ipilimumab 3 mg/kg every 3 weeks [4 doses] and then followed by nivolumab $240 \mathrm{mg}$ every 2 weeks), the objective response rate (ORR) was 32\% (95\% Cl: 20-47). In group B (obtaining nivolumab $1 \mathrm{mg} / \mathrm{kg}$ with ipilimumab $3 \mathrm{mg} / \mathrm{kg}$ every 3 weeks (4 doses)) ORR was 27\% (95\% Cl: 15-41) and in group C (with nivolumab 3 mg/kg every 2 weeks plus ipilimumab 1 mg/kg administration every 6 weeks) - 29\% (95\% Cl: 17-43). In groups $B$ and $C$, the median duration of response was, respectively: 15.2 months (4.2-29.9+) and 21.7 months (2.8-32.7+) while in group A it was not reached until the end of the study period and this therapeutic pattern was approved in the US concerning the results and the good safety profile [69].

\section{Tremelimumab and durvalumab}

According to the results of the NCT02519348 clinical trial, tremelimumab (anti-CTLA-4 antibody) administered in a single dose of $300 \mathrm{mg}$ combined with durvalumab (anti-PD-L1) in a dose of $1500 \mathrm{mg}$ showed promising clinical activity and tolerance in patients with HCC with median OS of 18.73 months (10.78-27.27) and median ORR of 24\% (95\% Cl: 14.9-35.3) with an acceptable safety profile. Tremelimumab-durvalumab combination remains evaluated in a phase III trial (HIMALAYA, NCT03298451) [70].

\section{Ramucirumab and durvalumab}

A 25-center study led by Bang [71] investigated the effectiveness of combined therapy with ramucirumab (IgG1, anti-VEGFR2) and durvalumab (IgG1, anti-PD-L1). The research subjects consisted of 28 patients diagnosed with HCC who had already been tre- 
ated before. In the course of the study it was possible to obtain a partial tumor response to treatment in 3 out of 28 patients, of which two showed high PD-L1 expression. In contrast, 24 patients experienced treatment-related adverse events (TRAE). The most common were diarrhea $(n=8)$, fatigue $(n=6)$ and increased blood pressure $(n=4)$. Two patients died during the study due to complications: acute hepatitis (TRAE) and acute respiratory distress syndrome (considered unrelated to treatment). It was assessed that the side effects of the combination of these two drugs do not go beyond the known complications of using each of them separately.

\section{Pembrolizumab and lenvatinib}

The effectiveness of the combination of pembrolizumab (anti-PD-1 antibodies) and lenvatinib (inhibitor of VEGFR1, VEGFR2 and VEGFR3 kinases) was investigated by Finn et al. [72]. A total of 100 patients received the combination of pembrolizumab and lenvatinib for an average of 7.5 months. Treatment efficacy was measured by the Modified Solid Tumor Response Criteria (mRECIST). The objective response rate (ORR) was 46.0\% (95\% Cl: 36.0-56.3). The median duration of response (DOR) was 8.6 months ( $95 \% \mathrm{Cl}: 6.9$ months to not estimable [NE]), the time to respond (TTR) - 1.9 months and PFS - 9.3 months (95\% Cl: 5.6-9.7 months). Median OS was 22 months ( $95 \% \mathrm{Cl}: 20.4$ months to NE). The shrinkage of the tumor size was observed in $89 \%$ of the subjects. Almost all patients (99\%) experienced side effects and the vast majority (95\%) reported more than one. The most common ones were: hypertension (36\%), diarrhea (35\%), fatigue (30\%), decreased appetite (28\%) and hypothyroidism (25\%). 13 patients died during the course of study while 3 of the deaths were considered treatment-related. This study also did not find any side effects that would be different from those of the two drugs administered alone.

While in the above study the combination of pembrolizumab and lenvatinib is used as the first-line treatment, there are isolated reports of patients successfully treated with combination therapy after using sorafenib [73] or pembrolizumab as monotherapy [74]. Not to mention the promising results of studies testing the effectiveness of this treatment in the therapy of endometrial cancer [75, 76], stomach [77] or kidney [78].

\section{Avelumab and axitinib}

Another variant of combination therapy is the combination of avelumab (anti-PD-L1 immunoglobulin) and axitinib (a selective VEGFR kinase inhibitor). This therapy has been shown to be effective in the treatment of kidney cancer $[79,80]$.

A study by Masatoshi Kudo et al. [81] involved 22 HCC patients who were administered these two drugs. A reduction in tumor size was noted (according to mRECIST) in 16 patients and ORR was 31.8\% (95\% Cl: 13.9-54.9). Side effects such as hypertension (50\% of the respondents), hypothyroidism (31.8\%), and hand-foot syndrome (22.7\%) were observed.

\section{Bevacizumab and erlotinib}

An alternative version of combination therapy is the combination of a VEGFR inhibitor with a drug that inhibits EGFR tyrosine kinase. A study verifying the effectiveness of such treatment was carried out by M. B. Thomas et al. [82]. 90 subjects with advanced HCC were randomized into two groups and treated with a combination of bevacizumab (anti-VEGFR immunoglobulin) and erlotinib ( $n=45$ ) or sorafenib in monotherapy $(n=45)$. The median OS was identical for both treatments and reached 8.5 months (95\% Cl: 7.00-13.9 for bevacizumab+erlotinib vs. 95\% Cl: 5.69-12.2 for sorafenib). However, the duration of event free survival (EFS) favored the combination treatment (median - 4.37 months, 95\% Cl: 2.99-7.36) over monotherapy (median - 2.76 months, 95\% Cl: 1.84-4.80). Side effects were more frequent in the sorafenib group but also in this group the treatment was discontinued much more often due to the occurrence of serious complications.

The researchers, led by Liyun He, also came to similar conclusions. Their study included 342 patients with HCC and showed that bevacizumab and erlotinib therapy is as effective as sorafenib therapy and is associated with lower toxicity and better tolerance by patients [83]. However, this combined therapy does not show significant efficacy in second-line treatment [84] or treatment of residual disease [85]. Table I shows sorafenib and second-line treatment options.

\section{Discussions}

Multi-kinase inhibitors and anti-angiogenic drugs have been the most commonly applied therapeutic options in HCC treatment, including sorafenib, as the treatment by choice in advanced HCC. Unfortunately, these options present certain disadvantages. Their high toxicity and frequent cases of disease progression as well as possible therapy-tolerance development cause a high number of failures in HCC therapy.

Among the drugs that can be used in HCC therapy after unsuccessful first-line treatment are lenvatinib, regorafenib, and cabozantinib, while tivantinib, brivanib, and everolimus were rejected in this regard. Regorafenib has proven effectiveness after previous treatment with sorafenib failure due to progression or in tolerance. Cabozantinib, in turn, is effective to some degree in patients with tumor progression.

However, disadvantages and limitations of therapy cause a pressing need for development of innovative therapeutic strategies in HCC treatment. Among the others, some immunotherapeutic agents are highly prospective.

A relatively high number of clinical trials and some meta-analyzes involved PD-1 inhibitors of immune checkpoints (PD-1, PD-L1), including nivolumab, camrelizumab, pembrolizumab, durvalumab, and tremelimumab. Existing data concerning immunotherapy indicate various efficacy, but good tolerance of most of the agents and their generally acceptable safety profile. So far, clinical trials of nivolumab show a longer median survival rate than in sorafenib groups, making the 
Table I. Overall response rate (ORR), overall survival (OS), time to progression (TTP) / progression-free survival (PFS) - sorafenib and second-line treatment options

\begin{tabular}{|c|c|c|c|c|}
\hline & ORR & Median OS (months) & Median TTP / PFS (months) & Citation \\
\hline \multirow[t]{2}{*}{ sorafenib } & & 10.7 (vs. 7.9 placebo) & 2.7 (TTP) & {$[10,11]$} \\
\hline & & 10.0 (vs. 15.2 sorafenib + cisplatin) & 3.9 (TTP) & [12] \\
\hline cabozantinib & & 10.2 (vs. 8.0 placebo) & 5.2 (PFS) & [27] \\
\hline \multirow[t]{2}{*}{ ramucirumab } & & 9.2 (vs. 7.6 placebo) & & \multirow{2}{*}{$\begin{array}{l}{[29]} \\
{[31]}\end{array}$} \\
\hline & & 8.5 (vs. 7.3 placebo) & 2.8 (PFS) & \\
\hline nivolumab & & 16.39 (vs. 15.69) & & {$[45,46]$} \\
\hline camrelizumab & & 13.8 & & [49] \\
\hline durvalumab & & 13.2 & & [55] \\
\hline tremelimumab & & 12.3 & 7.4 (PFS) & [66] \\
\hline atezolizumab + bevacizumab & & & 5.6 (PFS) & [63] \\
\hline atezolizumab + bevacizumab & & & 6.8 (PFS) & [64] \\
\hline nivolumab + ipilimumab & $27-32 \%$ & & & {$[65]$} \\
\hline tremelimumab + durvalumab & $24 \%$ & 18.73 & & [66] \\
\hline pembrolizumab + lenvatinib & $46 \%$ & & & [68] \\
\hline avelumab + axitinib & $31.8 \%$ & & & [77] \\
\hline bevacizumab + erlotinib & & 8.5 & & [78] \\
\hline
\end{tabular}

immunotherapeutic agents both a possible alternative for sorafenib and a therapeutic option for second-line treatment. Camrelizumab research, in turn, indicates a median survival rate shorter compared to nivolumab, but without serious adverse effects during the therapy.

Another group of therapies involve combinations of anti-angiogenesis drugs with inhibitors of immune checkpoints effective against lung cancer. Among them nivolumab and ipilimumab, tremelimumab and durvalumab, as well as ramucirumab and durvalumab combinations are characterized by at least an acceptable safety profile and tolerance, while neither the combinations of pembrolizumab with lenvatinib, nor ramucirumab with durvalumab show other side effects than the drugs used alone. Some of the combinations, including avelumab and axitinib have proven efficacy in tumor size reduction, while, bevacizumab and erlotinib combine treatment present duration of EFS better than monotherapy.

\section{Conclusions}

The promising results of cancer immunotherapy may offer new hope for patients diagnosed with HCC. In those with advanced cancer, some immunotherapeutic agents may be a safe and an effective alternative for chemotherapy; alternatively they can constitute medications to be applied as a part of second-line treatment after the failure of previous options mentioned earlier. Particularly good results have already been achieved in combined therapy clinical trials.
A serious weakness of the studies existing so far is that they are often based on isolated reports or have other crucial limitations. Only a few immunotherapeutic agents have been already approved or are undergoing the final stages of clinical trials, while others remain highly experimental. Research projects aiming to gain more clinical data concerning the efficacy and safety of both drugs used in monotherapies or in combined therapies are already underway.

\section{Conflict of interest: none declared}

\section{Katarzyna Szklener}

Medical University of Lublin

Department of Clinical Oncology and Chemotherapy

ul. Jaczewskiego 8

20-090 Lublin, Poland

e-mail:katarzyna.szklener@op.pl

\section{Received: 14 Aug 2021}

Accepted: 11 Nov 2021

\section{References}

1. Jemal A, Bray F, Center MM, et al. Global cancer statistics. CA Cancer J Clin. 2011;61(2):69-90, doi: 10.3322/caac.20107, indexed in Pubmed: 21296855.

2. Ozakyol A. Global Epidemiology of Hepatocellular Carcinoma (HCC Epidemiology). J Gastrointest Cancer. 2017; 48(3): 238-240, doi: 10.1007/ s12029-017-9959-0, indexed in Pubmed: 28626852.

3. El-Serag HB, Rudolph KL. Hepatocellular carcinoma: epidemiology and molecular carcinogenesis. Gastroenterology. 2007; 132(7): 2557-2576, doi: 10.1053/j.gastro.2007.04.061, indexed in Pubmed: 17570226.

4. Petruzziello A. Epidemiology of Hepatitis B Virus (HBV) and Hepatitis C Virus (HCV) Related Hepatocellular Carcinoma. Open Virol J. 2018; 
12: 26-32, doi: 10.2174/1874357901812010026, indexed in Pubmed: 29541276.

5. Perz JF, Armstrong GL, Farrington LA, et al. The contributions of hepatitis $B$ virus and hepatitis $C$ virus infections to cirrhosis and primary liver cancer worldwide. J Hepatol. 2006; 45(4): 529-538, doi: 10.1016/j. jhep.2006.05.013, indexed in Pubmed: 16879891.

6. Margini C, Dufour JF. The story of HCC in NAFLD: from epidemiology, across pathogenesis, to prevention and treatment. Liver Int. 2016; 36(3): 317-324, doi: 10.1111/liv.13031, indexed in Pubmed: 26601627.

7. Bruix J, Reig M, Sherman M. Evidence-Based Diagnosis, Staging, and Treatment of Patients With Hepatocellular Carcinoma. Gastroenterology. 2016; 150(4): 835-853, doi: 10.1053/j.gastro.2015.12.041, indexed in Pubmed: 26795574.

8. Escudier B, Worden F, Kudo M. Sorafenib: key lessons from over 10 years of experience. Expert Rev Anticancer Ther. 2019; 19(2): 177-189, doi: 10.1080/14737140.2019.1559058, indexed in Pubmed: 30575405.

9. Abdelgalil AA, Alkahtani HM, Al-Jenoobi FI. Sorafenib. Profiles Drug Subst Excip Relat Methodol. 2019; 44: 239-266, doi: 10.1016/ bs.podrm.2018.11.003, indexed in Pubmed: 31029219.

10. Llovet JM, Ricci S, Mazzaferro V, et al. SHARP Investigators Study Group. Sorafenib in advanced hepatocellular carcinoma. N Engl J Med. 2008; 359(4): 378-390, doi: 10.1056/NEJMoa0708857, indexed in Pubmed: 18650514.

11. Bruix J, Raoul JL, Sherman M, et al. Efficacy and safety of sorafenib in patients with advanced hepatocellular carcinoma: subanalyses of a phase III trial. J Hepatol. 2012; 57(4): 821-829, doi: 10.1016/j.jhep.2012.06.014, indexed in Pubmed: 22727733.

12. Kondo $\mathrm{M}$, Morimoto $\mathrm{M}$, Kobayashi $\mathrm{S}$, et al. Randomized, phase II trial of sequential hepatic arterial infusion chemotherapy and sorafenib versus sorafenib alone as initial therapy for advanced hepatocellular carcinoma: SCOOP-2 trial. BMC Cancer. 2019; 19(1): 954, doi: 10.1186/ s12885-019-6198-8, indexed in Pubmed: 31615466.

13. Al-Salama ZT, Syed YY, Scott LJ. Lenvatinib: A Review in Hepatocellular Carcinoma. Drugs. 2019; 79(6): 665-674, doi: 10.1007/s40265-01901116-x, indexed in Pubmed: 30993651.

14. Rimassa L, Wörns MA. Navigating the new landscape of second-line treatment in advanced hepatocellular carcinoma. Liver Int. 2020; 40(8): 1800-1811, doi: 10.1111/liv.14533, indexed in Pubmed: 32432830.

15. Fung A, Tam V, Meyers D, et al. Real world eligibility for cabozantinib (C), regorafenib (Reg), and ramucirumab (Ram) in hepatocellular carcinoma (HCC) patients after sorafenib (S). J Clin Oncol. 2019; 37(4_suppl): 422-422, doi: 10.1200/jco.2019.37.4_suppl.422.

16. Rimassa $L$, Assenat $E$, Peck-Radosavljevic $M$, et al. Tivantinib for second-line treatment of MET-high, advanced hepatocellular carcinoma (METIV-HCC): a final analysis of a phase 3, randomised, placebo-controlled study. Lancet Oncol. 2018; 19(5): 682-693, doi: 10.1016/S14702045(18)30146-3, indexed in Pubmed: 29625879.

17. Llovet JM, Decaens T, Raoul JL, et al. Brivanib in patients with advanced hepatocellular carcinoma who were intolerant to sorafenib or for whom sorafenib failed: results from the randomized phase III BRISK-PS study. J Clin Oncol. 2013; 31(28): 3509-3516, doi: 10.1200/JCO.2012.47.3009, indexed in Pubmed: 23980090.

18. Zhu AX, Kudo M, Assenat $E$, et al. Effect of everolimus on survival in advanced hepatocellular carcinoma after failure of sorafenib: the EVOLVE-1 randomized clinical trial. JAMA. 2014; 312(1): 57-67, doi: 10.1001/jama.2014.7189, indexed in Pubmed: 25058218.

19. Bruix J, Qin S, Merle P, et al. Regorafenib for patients with hepatocelIular carcinoma who progressed on sorafenib treatment (RESORCE): a randomised, double-blind, placebo-controlled, phase 3 trial. The Lancet. 2017; 389(10064): 56-66, doi: 10.1016/s0140-6736(16)32453-9.

20. Grothey A, Blay JY, Pavlakis N, et al. Evolving role of regorafenib for the treatment of advanced cancers. Cancer Treat Rev. 2020; 86: 101993, doi: 10.1016/j.ctrv.2020.101993, indexed in Pubmed: 32199197.

21. Abou-Elkacem L, Arns S, Brix G, et al. Regorafenib inhibits growth, angiogenesis, and metastasis in a highly aggressive, orthotopic colon cancer model. Mol Cancer Ther. 2013; 12(7): 1322-1331, doi: 10.1158/15357163.MCT-12-1162, indexed in Pubmed: 23619301.

22. Yakes FM, Chen J, Tan J, et al. Cabozantinib (XL184), a novel MET and VEGFR2 inhibitor, simultaneously suppresses metastasis, angiogenesis, and tumor growth. Mol Cancer Ther. 2011; 10(12): 2298-2308, doi: 10.1158/1535-7163.MCT-11-0264, indexed in Pubmed: 21926191.

23. Birchmeier C, Birchmeier W, Gherardi E, et al. Met, metastasis, motility and more. Nat Rev Mol Cell Biol. 2003; 4(12): 915-925, doi: 10.1038/ nrm1261, indexed in Pubmed: 14685170.

24. Michieli P, Mazzone M, Basilico C, et al. Targeting the tumor and its microenvironment by a dual-function decoy Met receptor. Cancer
Cell. 2004; 6(1): 61-73, doi: 10.1016/j.ccr.2004.05.032, indexed in Pubmed: 15261142.

25. Abounader R, Lal B, Luddy $C$, et al. In vivo targeting of SF/HGF and c-met expression via U1snRNA/ribozymes inhibits glioma growth and angiogenesis and promotes apoptosis. FASEB J. 2002; 16(1): 108-110, doi: 10.1096/fj.01-0421fje, indexed in Pubmed: 11729097.

26. Firtina Karagonlar Z, Koc D, Iscan E, et al. Elevated hepatocyte growth factor expression as an autocrine c-Met activation mechanism in acquired resistance to sorafenib in hepatocellular carcinoma cells. Cancer Sci. 2016; 107(4): 407-416, doi: 10.1111/cas.12891, indexed in Pubmed: 26790028.

27. Abou-Alfa GK, Meyer T, Cheng AL, et al. Cabozantinib in Patients with Advanced and Progressing Hepatocellular Carcinoma. N Engl J Med. 2018; 379(1): 54-63, doi: 10.1056/NEJMoa1717002, indexed in Pubmed: 29972759.

28. Spratlin JL, Cohen RB, Eadens M, et al. Phase I pharmacologic and biologic study of ramucirumab (IMC-1121B), a fully human immunoglobulin G1 monoclonal antibody targeting the vascular endothelial growth factor receptor-2. J Clin Oncol. 2010; 28(5): 780-787, doi: 10.1200/ JCO.2009.23.7537, indexed in Pubmed: 20048182.

29. Zhu AX, Park JOh, Ryoo BY, et al. REACHTrial Investigators. Ramucirumab versus placebo as second-line treatment in patients with advanced hepatocellular carcinoma following first-line therapy with sorafenib (REACH): a randomised, double-blind, multicentre, phase 3 trial. Lancet Oncol. 2015; 16(7): 859-870, doi: 10.1016/S1470-2045(15)00050-9, indexed in Pubmed: 26095784.

30. Zhu A, Ryoo BY, Yen CJ, et al. Ramucirumab (RAM) as second-line treatment in patients (pts) with advanced hepatocellular carcinoma (HCC): Analysis of patients with elevated a-fetoprotein (AFP) from the randomized phase III REACH study. J Clin Oncol. 2015; 33(3 suppl): 232-232, doi: 10.1200/jco.2015.33.3_suppl.232.

31. Zhu AX, Kang YK, Yen CJ, et al. REACH-2 study investigators. Ramucirumab after sorafenib in patients with advanced hepatocellular carcinoma and increased a-fetoprotein concentrations (REACH-2): a randomised, double-blind, placebo-controlled, phase 3 trial. Lancet Oncol. 2019; 20(2): 282-296, doi: 10.1016/S1470-2045(18)30937-9, indexed in Pubmed: 30665869.

32. Johnston MP, Khakoo SI. Immunotherapy for hepatocellular carcinoma: Current and future. World J Gastroenterol. 2019; 25(24): 2977-2989, doi: 10.3748/wjg.v25.i24.2977, indexed in Pubmed: 31293335.

33. Ishiguro T, Sano Y, Komatsu SI, et al. An anti-glypican 3/CD3 bispecific T cell-redirecting antibody for treatment of solid tumors. Sci Transl Med. 2017; 9(410), doi: 10.1126/scitransImed.aal4291, indexed in Pubmed: 28978751.

34. Takahashi Y, Ohta T, Mai M. Angiogenesis of AFP producing gastric carcinoma: correlation with frequent liver metastasis and its inhibition by anti-AFP antibody. Oncol Rep. 2004; 11(4): 809-813, indexed in Pubmed: 15010877.

35. Ghassemi S, Milone MC. Manufacturing Chimeric Antigen Receptor (CAR) T Cells for Adoptive Immunotherapy. J Vis Exp. 2019(154), doi: 10.3791/59949, indexed in Pubmed: 31904011.

36. Mata-Molanes JJ, Sureda González M, Valenzuela Jiménez B, et al. Cancer Immunotherapy with Cytokine-Induced Killer Cells. Target Oncol. 2017; 12(3): 289-299, doi: 10.1007/s11523-017-0489-2, indexed in Pubmed: 28474278.

37. Bagheri Y, Barati A, Aghebati-Maleki A, et al. Current progress in cancer immunotherapy based on natural killer cells. Cell Biol Int. 2021; 45(1): 2-17, doi: 10.1002/cbin.11465, indexed in Pubmed: 32910474.

38. Calmeiro J, Carrascal MA, Tavares AR, et al. Dendritic Cell Vaccines for Cancer Immunotherapy: The Role of Human Conventional Type 1 Dendritic Cells. Pharmaceutics. 2020; 12(2), doi: 10.3390/pharmaceutics12020158, indexed in Pubmed: 32075343.

39. Slingluff $C$. The Present and Future of Peptide Vaccines for Cancer. The Cancer Journal. 2011; 17(5): 343-350, doi: 10.1097/ppo.0b013e$318233 \mathrm{e} 5 \mathrm{~b} 2$.

40. Nguyen LT, Ohashi PS. Clinical blockade of PD1 and LAG3--potential mechanisms of action. Nat Rev Immunol. 2015; 15(1): 45-56, doi: 10.1038/nri3790, indexed in Pubmed: 25534622.

41. Hato T, Goyal L, Greten TF, et al. Immune checkpoint blockade in hepatocellular carcinoma: current progress and future directions. Hepatology. 2014;60(5): 1776-1782, doi: 10.1002/hep.27246, indexed in Pubmed: 24912948.

42. Fife BT, Pauken KE. The role of the PD-1 pathway in autoimmunity and peripheral tolerance. Ann N Y Acad Sci. 2011; 1217: 45-59, doi: 10.1111/j.1749-6632.2010.05919.x, indexed in Pubmed: 21276005.

43. Hui E, Cheung J, Zhu J, et al. T cell costimulatory receptor CD28 is a primary target for PD-1-mediated inhibition. Science. 2017; 
355(6332): 1428-1433, doi: 10.1126/science.aaf1292, indexed in Pubmed: 28280247.

44. Han Y, Liu D, Li L. PD-1/PD-L1 pathway: current researches in cancer. Am J Cancer Res. 2020; 10(3): 727-742, indexed in Pubmed: 32266087.

45. El-Khoueiry A, Sangro B, Yau T, et al. Nivolumab in patients with advanced hepatocellular carcinoma (CheckMate 040): an open-label, non-comparative, phase 1/2 dose escalation and expansion trial. The Lancet. 2017; 389(10088): 2492-2502, doi: 10.1016/s0140-6736(17)31046-2.

46. Bristol-Myers Squibb. A Randomized, Multi-center Phase III Study of Nivolumab Versus Sorafenib as First-Line Treatment in Patients With Advanced Hepatocellular Carcinoma (CheckMate 459: CHECKpoint Pathway and nivoluMAb Clinical Trial Evaluation 459). Report No.: NCT02576509. https://clinicaltrials.gov/ct2/show/NCT02576509 (10.05.2021).

47. Mo H, Huang J, $\mathrm{Xu}$ J, et al. Safety, anti-tumour activity, and pharmacokinetics of fixed-dose SHR-1210, an anti-PD-1 antibody in advanced solid tumours: a dose-escalation, phase 1 study. Br J Cancer. 2018; 119(5): 538-545, doi: 10.1038/s41416-018-0100-3, indexed in Pubmed: 29755117

48. Qin S, Ren Z, Meng Z, et al. Camrelizumab in patients with previously treated advanced hepatocellular carcinoma: a multicentre, open-label, parallel-group, randomised, phase 2 trial. Lancet Oncol. 2020; 21(4): 571-580, doi: 10.1016/S1470-2045(20)30011-5, indexed in Pubmed: 32112738 .

49. Jiangsu HengRui Medicine Co., Ltd. A Phase III, Multicentered, Randomized, Double-blinded, Parallel Controlled Study to Evaluate Camrelizumab (PD1 Antibody) in Combination With FOLFOX4 Regimen Versus Placebo in Combination With FOLFOX4 Regime in First-Line Therapy in Subjects With Advanced Hepatocellular Carcinoma (HCC). Report No.: NCT03605706. https://clinicaltrials.gov/ct2/show/NCT03605706 (10.05.2021).

50. Finn RS, Ryoo BY, Merle P, et al. KEYNOTE-240 investigators. Pembrolizumab As Second-Line Therapy in Patients With Advanced Hepatocellular Carcinoma in KEYNOTE-240: A Randomized, Double-Blind, Phase IIITrial. J Clin Oncol. 2020; 38(3): 193-202, doi: 10.1200/JCO.19.01307, indexed in Pubmed: 31790344.

51. Zhu AX, Finn RS, Edeline J, et al. KEYNOTE-224 investigators. Pembrolizumab in patients with advanced hepatocellular carcinoma previously treated with sorafenib (KEYNOTE-224): a non-randomised, open-label phase 2 trial. Lancet Oncol. 2018; 19(7): 940-952, doi: 10.1016/S14702045(18)30351-6, indexed in Pubmed: 29875066.

52. Innovent Biologics (Suzhou) Co. Ltd. A Randomized, Open-label $\square$ Multicenter Study to Evaluate the Efficacy and Safety of the Combination of Sintilimab and IBI305 Compared to Sorafenib in the First-Line Treatment of Patients With Advanced Hepatocellular Carcinoma. (ORIENT-32). Report No.: NCT03794440. https://clinicaltrials.gov/ct2/ show/NCT03794440 (10.05.2021).

53. BeiGene. RATIONALE-301: A Randomized, Open-label, Multicenter Phase 3 Study to Compare the Efficacy and Safety of BGB-A317 Versus Sorafenib as First-Line Treatment in Patients With Unresectable Hepatocellular Carcinoma. Report No.: NCT03412773. https://clinicaltrials. gov/ct2/show/NCT03412773 (10.05.2021).

54. Voutsadakis IA. PD-1 inhibitors monotherapy in hepatocellular carcinoma: Meta-analysis and systematic review. Hepatobiliary Pancreat Dis Int. 2019; 18(6): 505-510, doi: 10.1016/j.hbpd.2019.09.007, indexed in Pubmed: 31551142.

55. Wainberg Z, Segal N, Jaeger D, et al. Safety and clinical activity of durvalumab monotherapy in patients with hepatocellular carcinoma (HCC). J Clin Oncol. 2017; 35(15_suppl): 4071-4071, doi: 10.1200/ jco.2017.35.15_suppl.4071.

56. Kroemer G, Zitvogel L, Krummel MF, et al. CD28 and CTLA-4 have opposing effects on the response of T cells to stimulation. J Exp Med. 1995; 182(2): 459-465, doi: 10.1084/jem.182.2.459, indexed in Pubmed: 7543139

57. Chen J, Ganguly A, Mucsi AD, et al. Strong adhesion by regulatory T cells induces dendritic cell cytoskeletal polarization and contact-dependent lethargy. J Exp Med. 2017; 214(2): 327-338, doi: 10.1084/jem.20160620, indexed in Pubmed: 28082358.

58. Sangro B, Gomez-Martin C, de la Mata M, et al. A clinical trial of CTLA-4 blockade with tremelimumab in patients with hepatocellular carcinoma and chronic hepatitis C. J Hepatol. 2013; 59(1): 81-88, doi: 10.1016/j. jhep.2013.02.022, indexed in Pubmed: 23466307.

59. AstraZeneca. A Randomized, Open-label, Multi-center Phase III Study of Durvalumab and Tremelimumab as First-line Treatment in Patients With Advanced Hepatocellular Carcinoma [Internet]. Report No.: NCT03298451. https://clinicaltrials.gov/ct2/show/NCT03298451 (10.05.2021).
60. Novartis Pharmaceuticals. A Phase I/lb, Open-label, Multi-center Dose Escalation Study of NIS793 in Combination With PDR001 in Adult Patients With Advanced Malignancies. Report No.: NCT02947165. https:// clinicaltrials.gov/ct2/show/NCT02947165 (10.05.2021).

61. University of Hawaii. Phase II Study of TSR-022 in Combination With TSR042 for the Treatment of Advanced Hepatocellular Carcinoma. Report No.: NCT03680508. https://clinicaltrials.gov/ct2/show/NCT03680508 (10.05.2021).

62. Bristol-Myers Squibb. A Phase I/2a Dose Escalation and Cohort Expansion Study of the Safety, Tolerability, and Efficacy of Anti-LAG-3 Monoclonal Antibody (BMS-986016) Administered Alone and in Combination With Anti-PD-1 Monoclonal Antibody (Nivolumab, BMS-936558) in Advanced Solid Tumors. Report No.: NCT01968109. https://clinicaltrials. gov/ct2/show/NCT01968109 (10.05.2021).

63. Nobuoka D, Motomura Y, Shirakawa $\mathrm{H}$, et al. Radiofrequency ablation for hepatocellular carcinoma induces glypican-3 peptide-specific cytotoxic T lymphocytes. Int J Oncol. 2012; 40(1): 63-70, doi: 10.3892/ ijo.2011.1202, indexed in Pubmed: 21922136.

64. Zerbini A, Pilli M, Laccabue $D$, et al. Radiofrequency thermal ablation for hepatocellular carcinoma stimulates autologous NK-cell response. Gastroenterology. 2010; 138(5): 1931-1942, doi: 10.1053/j. gastro.2009.12.051, indexed in Pubmed: 20060829.

65. Hansler J, Wissniowski TT, Schuppan D, et al. Activation and dramatically increased cytolytic activity of tumor specific T lymphocytes after radio-frequency ablation in patients with hepatocellular carcinoma and colorectal liver metastases. World J Gastroenterol. 2006; 12(23): 3716-3721, doi: 10.3748/wjg.v12.i23.3716, indexed in Pubmed: 16773688.

66. Duffy AG, Ulahannan SV, Makorova-Rusher O, et al. Tremelimumab in combination with ablation in patients with advanced hepatocellular carcinoma. J Hepatol. 2017; 66(3): 545-551, doi: 10.1016/j. jhep.2016.10.029, indexed in Pubmed: 27816492.

67. Lee MS, Ryoo BY, Hsu CH, et al. GO30140 investigators. Atezolizumab with or without bevacizumab in unresectable hepatocellular carcinoma (GO30140): an open-label, multicentre, phase 1b study. Lancet Oncol. 2020; 21(6): 808-820, doi: 10.1016/S1470-2045(20)30156-X, indexed in Pubmed: 32502443.

68. Hack SP, Spahn J, Chen M, et al. IMbrave 050: a Phase III trial of atezolizumab plus bevacizumab in high-risk hepatocellular carcinoma after curative resection or ablation. Future Oncol. 2020; 16(15): 975-989, doi: 10.2217/fon-2020-0162, indexed in Pubmed: 32352320.

69. Yau T, Kang YK, Kim TY, et al. Efficacy and Safety of Nivolumab Plus Ipilimumab in Patients With Advanced Hepatocellular Carcinoma Previously Treated With Sorafenib: The CheckMate 040 Randomized Clinical Trial. JAMA Oncol. 2020; 6(11): e204564, doi: 10.1001/jamaoncol.2020.4564, indexed in Pubmed: 33001135.

70. Imfinzi plus tremelimumab demonstrated promising clinical activity and tolerability in patients with advanced liver cancer. https://www. astrazeneca.com/media-centre/press-releases/2020/imfinzi-plustremelimumab-demonstrated-promising-clinical-activity-andtolerability-in-patients-with-advanced-liver-cancer.html (01.03.2021).

71. Bang YJ, Golan T, Dahan L, et al. Ramucirumab and durvalumab for previously treated, advanced non-small-cell lung cancer, gastric/gastro-oesophageal junction adenocarcinoma, or hepatocellular carcinoma: An open-label, phase la/b study (JVDJ). Eur J Cancer. 2020; 137: 272-284, doi: 10.1016/j.ejca.2020.06.007, indexed in Pubmed: 32827847.

72. Finn RS, Ikeda M, Zhu AX, et al. Phase Ib Study of Lenvatinib Plus Pembrolizumab in Patients With Unresectable Hepatocellular Carcinoma. J Clin Oncol. 2020; 38(26): 2960-2970, doi: 10.1200/JCO.20.00808, indexed in Pubmed: 32716739.

73. Schulte N, Li M, Zhan T, et al. Response of advanced HCC to pembrolizumab and lenvatinib combination therapy despite monotherapy failure. Z Gastroenterol. 2020; 58(8): 773-777, doi: 10.1055/a-1190-5681, indexed in Pubmed: 32785913.

74. Liu Z, Li X, He X, et al. Complete response to the combination of Lenvatinib and Pembrolizumab in an advanced hepatocellular carcinoma patient: a case report. BMC Cancer. 2019; 19(1): 1062, doi: 10.1186/ s12885-019-6287-8, indexed in Pubmed: 31703571.

75. Makker V, Taylor MH, Aghajanian C, et al. Lenvatinib Plus Pembrolizumab in Patients With Advanced Endometrial Cancer. J Clin Oncol. 2020; 38(26): 2981-2992, doi: 10.1200/JCO.19.02627, indexed in Pubmed: 32167863.

76. Makker V, Rasco D, Vogelzang NJ, et al. Lenvatinib plus pembrolizumab in patients with advanced endometrial cancer: an interim analysis of a multicentre, open-label, single-arm, phase 2 trial. Lancet Oncol. 2019; 20(5): 711-718, doi: 10.1016/S1470-2045(19)30020-8, indexed in Pubmed: 30922731. 
77. Kawazoe A, Fukuoka S, Nakamura Y, et al. Lenvatinib plus pembrolizumab in patients with advanced gastric cancer in the first-line or second-line setting (EPOC1706): an open-label, single-arm, phase 2 trial. Lancet Oncol. 2020; 21(8): 1057-1065, doi: 10.1016/S1470-2045(20)30271-0, indexed in Pubmed: 32589866.

78. Motzer R, Alekseev B, Rha SY, et al. CLEARTrial Investigators. Lenvatinib plus Pembrolizumab or Everolimus for Advanced Renal Cell Carcinoma. N Engl J Med. 2021; 384(14): 1289-1300, doi: 10.1056/NEJMoa2035716, indexed in Pubmed: 33616314.

79. Choueiri TK, Motzer RJ, Rini BI, et al. Updated efficacy results from the JAVELIN Renal 101 trial: first-line avelumab plus axitinib versus sunitinib in patients with advanced renal cell carcinoma. Ann Oncol. 2020; 31(8): 1030-1039, doi: 10.1016/j.annonc.2020.04.010, indexed in Pubmed: 32339648.

80. Motzer RJ, Penkov K, Haanen J, et al. Avelumab plus Axitinib versus Sunitinib for Advanced Renal-Cell Carcinoma. N Engl J Med. 2019; 380(12): 1103-1115, doi: 10.1056/NEJMoa1816047, indexed in Pubmed: 30779531.

81. Kudo $M$, Motomura $K$, Wada $Y$, et al. First-line avelumab + axitinib in patients with advanced hepatocellular carcinoma: Results from a phase 1b trial (VEGF Liver 100). J Clin Oncol. 2019; 37(15 suppl): 4072-4072, doi: 10.1200/jco.2019.37.15_suppl.4072.

82. Thomas MB, Garrett-Mayer E, Anis M, et al. A Randomized Phase II Open-Label Multi-Institution Study of the Combination of Bevacizumab and Erlotinib Compared to Sorafenib in the First-Line Treatment of Patients with Advanced Hepatocellular Carcinoma. Oncology. 2018; 94(6): 329-339, doi: 10.1159/000485384, indexed in Pubmed: 29719302.

83. He L, Deng $\mathrm{H}$, Lei J, et al. Efficacy of bevacizumab combined with erlotinib for advanced hepatocellular carcinoma: a single-arm meta-analysis based on prospective studies. BMC Cancer. 2019; 19(1): 276, doi: 10.1186/s12885-019-5487-6, indexed in Pubmed: 30922256.

84. Kaseb AO, Morris JS, Iwasaki M, et al. Phase II trial of bevacizumab and erlotinib as a second-line therapy for advanced hepatocellular carcinoma. Onco Targets Ther. 2016; 9: 773-780, doi: 10.2147/OTT.S91977, indexed in Pubmed: 26929648.

85. Yau T, Wong $\mathrm{H}, \mathrm{Chan} \mathrm{P}$, et al. Phase ll study of bevacizumab and erlotinib in the treatment of advanced hepatocellular carcinoma patients with sorafenib-refractory disease. Invest New Drugs. 2012; 30(6): 2384-2390, doi: 10.1007/s10637-012-9808-8, indexed in Pubmed: 22402942. 